\title{
Familial Presentation of Moyamoya Disease in India
}

\section{Siddharth Maheshwari ${ }^{1 *}$, Monali Chaturvedi ${ }^{2}$, Suman Kushwaha ${ }^{1}$ and Aldrin Anthony ${ }^{1}$}

${ }^{1}$ Department of Neurology, IHBAS (Institute of Human Behaviour and Allied

Sciences), New Delhi, India

${ }^{2}$ Department of Neuroradiology, IHBAS (Institute of Human Behaviour and Allied

Sciences), New Delhi, India

*Corresponding Author: Siddharth Maheshwari, Assistant Professor, Department

of Neurology, IHBAS (Institute of Human Behaviour and Allied Sciences), New Delhi, India.
Received: March 11, 2021

Published: April 10, 2021

(C) All rights are reserved by Siddharth

Maheshwari., et al.

\begin{abstract}
Moyamoya disease is a chronic progressive arteriopathy involving the cerebral vessels and is one of the rare causes of young stroke. Etiology can be idiopathic as well secondary to some other primary disease. It does affect the members of the same family but the definite pattern of inheritance has not been found out yet. There are few studies in the literature about the familial presentation of idiopathic Moyamoya disease, most of them from East Asian countries, which is why there are no common opinions and guidelines regarding the follow up and management of asymptomatic family members of such patients. We present and discuss the case reports of two family members with Moyamoya disease from India to further emphasise upon the need of research and long term follow up studies.
\end{abstract}

Keywords: Cerebral Arterial Diseases; Infarct; Intracranial Arterial Diseases; Movement Disorder; Moyamoya Disease

\section{Introduction}

Moyamoya disease one of the rare causes of young stroke is a chronic progressive arteriopathy involving the vessels of cerebrum. It does affect the members of the same family but without any definite pattern of inheritance . ,It is a rare cause of non-atherosclerotic cerebral vasculopathy, mostly prevalent in the Asian countries. Moyamoya disease manifestations in children are mostly secondary to occlusion of cerebral arteries resulting in neurological deficits.

Approximately 5 - $10 \%$ of the cases have found to be familial. Only few studies in literature about the familial occurrence of idiopathic Moyamoya disease, mostly from East Asian countries

\section{Case Reports}

Case 1

A 15-year-old boy presented with weakness of left upper and lower limbs for the past three months and multiple episodes of seizures for the past one year. There were no complaints of headache, dizziness, ear discharge, fever and trauma. Neurological examinations revealed power of $4 / 5$, increased muscle tone and exaggerated deep tendon reflexes with extensor plantar reflex left limbs. The patient was responsive, following oral commands with no speech or vision impairment and no signs of meningeal irritation. There was no history of tuberculosis or any other chronic disease in the past. No radiological evidence of tuberculosis was found. The laboratory evaluation was unremarkable for haematocrit, clotting time and sickling test. 
Imaging findings

Chest radiograph, echocardiography and bilateral carotid and vertebral artery Doppler were unremarkable for any pathology. Computed tomography (CT) scan revealed chronic infarcts and cerebral atrophy involving the right frontal and temporal lobes in the right middle cerebral artery territory with dilatation of left lateral ventricle (Figure 1a and 1b). CT angiography study (Figure 1c and 1d) depicted attenuated calibre of bilateral terminal internal carotid arteries (ICA), middle cerebral arteries (MCA) and anterior cerebral arteries (ACA). Multiple parenchymal and leptomeningeal collaterals were seen from the posterior circulation and transdural vessel branches of the external carotid circulation. The appearance of the collaterals was typical of the "puff of smoke" that has been defined for Moyamoya disease. In view of absence of clinical and radiological evidence of any other disease, the findings were found consistent with the diagnosis of Moyamoya disease.
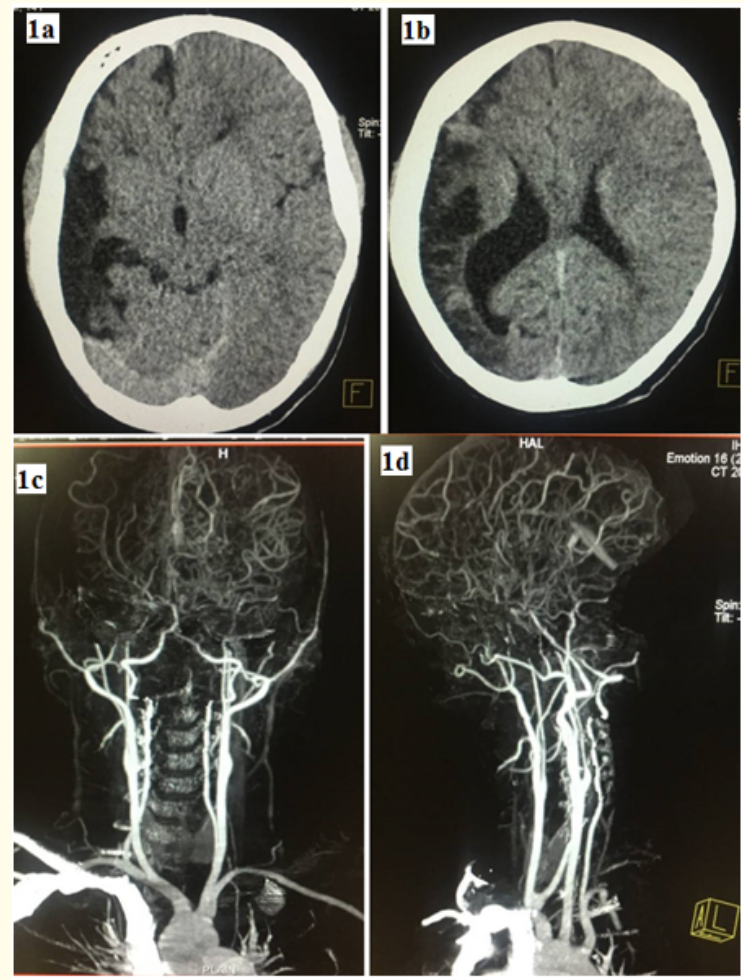

Figure 1: (a, b) Plain CT revealed chronic infarct and cerebral atrophy involving right frontal and parietal lobes with dilated right lateral ventricle. (c, d) CT angiogram depicted narrowing of bilateral terminal ICA and proximal ACA and MCA with multiple basal collaterals resembling puff of cigarette smoke.
Case 2

36-year-old male, father of the above case, presents with the complaint of right hemiparesis for the past 15 years. Present or past history was negative for any other disease. Signs of any other neurological deficit were absent. Routine blood examination and thrombotic parameters were within normal limits.

\section{Imaging findings}

Chest radiograph, echocardiogram and bilateral carotid-vertebral Doppler were unremarkable for any pathology. CT scan showed chronic infarct involving the left corona radiata (Figure 2a and $2 \mathrm{~b}$ ). CT angiography (Figure $2 \mathrm{c}$ and $2 \mathrm{~d}$ ) revealed narrowing of bilateral ICA and proximal MCA and ACA. Volume rendered 3D CT angiogram showed stenosis of terminal ICA with basal collaterals (Figure 3). Few basal and leptomeningeal collaterals were seen. Findings were consistent with the diagnosis of Moyamoya disease.

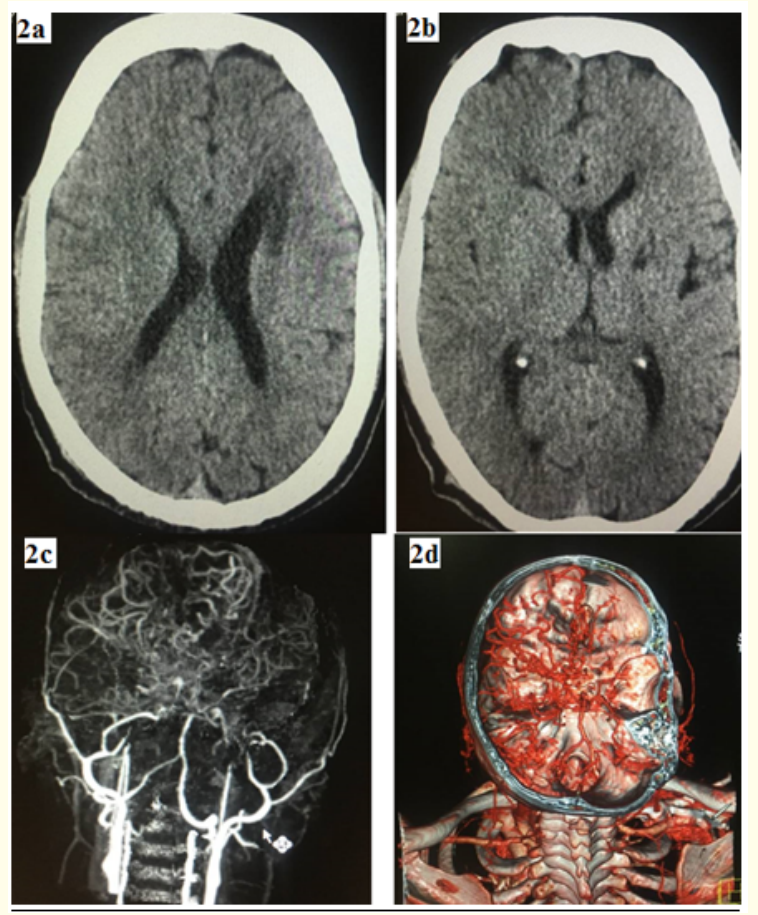

Figure 2: $(\mathrm{a}, \mathrm{b})$ Plain CT revealed chronic infarct and cerebral atrophy involving right frontal and parietal lobes with dilated right lateral ventricle. (c, d)Multiple basal are demonstrated in CT angiogram and volume rendered 3D CT angiogram. 

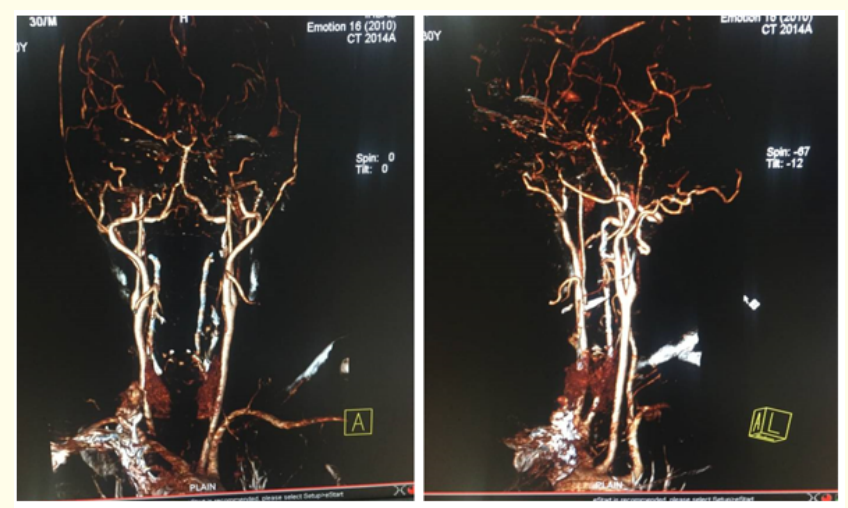

Figure 3: Volume rendered 3D CT angiogram showed stenosis of terminal ICA with basal collaterals.

\section{Discussion}

Moyamoya disease, a rare cause of non-atherosclerotic cerebral vasculopathy, mostly prevalent (10.5 patients per 100000 people) in the Asian countries [1]. Moyamoya disease manifestations in children are mostly secondary to occlusion of cerebral arteries resulting in recurrent hemiparesis, sensory/visual or speech impairment, transient ischemic attacks and intellectual deficits. Manifestations in adults are usually secondary to haemorrhagic stroke due to rupture of dilated fragile collaterals.

Approximately $5-10 \%$ of the cases have found to be familial. Different studies in the literature have suggested various Mendelian patterns of inheritance. Y Mineharu., et al. in their pedigree analysis of Japanese families found the mode of inheritance to be autosomal dominant with reduced penetrance [2]. Hidetoshi Ikeda., et al. in their study suggested the cause most probably be the polygenic [3].

Moyamoya is characterized by stenosis of distal internal carotid arteries and proximal anterior and middle cerebral arteries leading to the formation of extensive collaterals which appears as "puff of cigarette smoke drifted in the air" on imaging [4]. This characteristic angiographic pattern has been described as "Moyamoya phenomenon" [5]. "Moyamoya disease" term is used when the involvement is bilateral and there is no other cause. However, when associated with other neurological or extra neurological entities, term "Moyamoya syndrome" is used [5]. Along with clinical features, imaging plays a crucial role in moyamoya disease investigating the vasculopathy in craniocervical vessels, exploring the internal carotids, circle of Willis and collaterals to localize the cause.

Management of moyamoya involves measures to improve circulation and to control intracerebral oedema. Surgical revascularization to bypass the occlusion is achieved by anastomoses with branches of the external carotid artery. However, it does not hold true for all age groups. The disease is more progressive in the paediatric age group than the adults, making surgical revascularization the choice of treatment in former [6].

\section{Conclusion}

To the best of our knowledge, this is the first case report from India with the familial presentation of Moyamoya disease. our experience with these cases impresses upon the need for screening of family members of Moyamoya disease patients. The screening and early detection of disease in asymptomatic family members of Moyamoya disease patients can prevent the development of serious complications.

\section{Bibliography}

1. Baba T., et al. "Novel epidemiological features of moyamoya disease". Journal of Neurology, Neurosurgery, and Psychiatry 79.8 (2008): 900-904.

2. Mineharu Y., et al. "Inheritance pattern of familial moyamoya disease: autosomal dominant mode and genomic imprinting". Journal of Neurology, Neurosurgery and amp; Psychiatry 77.9 (2006): 1025-1029.

3. Ikeda H., et al. "Mapping of a Familial Moyamoya Disease Gene to Chromosome 3p24.2-p26". The American Journal of Human Genetics 64.2 (1999): 533-537.

4. Suzuki J and Takaku A. “Cerebrovascular “Moyamoya” Disease: Disease Showing Abnormal Net-Like Vessels in Base of Brain". JAMA Neurology 20.3 (1969): 288-299.

5. Guey S., et al. "Moyamoya disease and syndromes: from genetics to clinical management". The Application of Clinical Genetics 8 (2015): 49-68. 
6. Bao XY., et al. "Clinical Features, Surgical Treatment, and LongTerm Outcome in Pediatric Patients with Moyamoya Disease in China". Cerebrovascular Diseases 39.2 (2015): 75-81.

\section{Assets from publication with us}

- Prompt Acknowledgement after receiving the article

- Thorough Double blinded peer review

- Rapid Publication

- Issue of Publication Certificate

- High visibility of your Published work

Website: www.actascientific.com/

Submit Article: www.actascientific.com/submission.php

Email us: editor@actascientific.com

Contact us: +919182824667 